\title{
A NEW PROOF FOR THE HOSAY-LININGER THEOREM ABOUT CRUMPLED CUBES ${ }^{1}$
}

ROBERT J. DAVERMAN

I. Introduction. In 1963 Hosay [2] and Lininger [3], working independently, proved the following theorem:

Theorem. If $C$ is a crumpled cube in $S^{3}$, and $\epsilon$ is a positive number, then there is an $\epsilon$-homeomorphism $h$ from $C$ into $S^{3}$ such that the closure of $S^{3}-h(C)$ is a 3-cell.

Recently F. M. Lister [4] established a stronger version of Bing's Side Approximation Theorem. With this new result one can avoid certain complications encountered by Hosay and Lininger. The goal of this paper is to provide a short proof for their theorem, using this result of Lister. We understand that Robert F. Craggs has obtained a similar short proof.

Approximation Theorem (LiSTER). If $S$ is a 2-sphere in $E^{3}$, and $\epsilon$ is a positive number, then there exist an $\epsilon$-homeomorphism $\mathrm{g}$ from $S$ into $E^{3}$ and a collection of mutually exclusive $\epsilon$-disks $\left\{D_{i}\right\}_{i=1}^{m}$ on $S$ satisfying

(1) $g(S)$ is polyhedral,

(2) $g(S)-\bigcup_{i=1}^{m} g\left(\right.$ Int $\left.D_{i}\right)=g\left(S-\bigcup_{i=1}^{m}\right.$ Int $\left.D_{i}\right) \subset$ Ext $S$,

(3) $S-\bigcup_{i=1}^{m}$ Int $D_{i} \subset$ Int $g(S)$, and

(4) $g(S) \cap D_{i}=g\left(D_{i}\right) \cap D_{i}(i=1, \cdots, m)$.

A crumpled cube is a space homeomorphic to the union of some 2 -sphere in $E^{3}$ and its bounded complementary domain. For explanation of other terms and notations used here, the reader may see [3].

\section{Re-embedding crumpled cubes.}

Lemma. Suppose $C$ is a crumpled cube in $E^{3}$ with boundary $S$, and $\epsilon$ is a positive number. Then there exist an $\epsilon$-homeomorphism $h^{\prime}$ from $C$ into $E^{3}$ and a polyhedral 2-sphere $S^{\prime}$ homeomorphically within $\epsilon$ of $S$ such that $h^{\prime}(C) \subset$ Int $S^{\prime}$.

Proof. Using the Approximation Theorem for $\epsilon^{\prime}=\epsilon / 9$, we find a polyhedral 2-sphere $g(S)$, homeomorphically within $\epsilon^{\prime}$ of $S$, and a

Received by the editors February 25, 1968.

1 The material in this paper was included in the author's Ph.D. dissertation at the University of Wisconsin, written under the direction of R. H. Bing. Research was supported by NSF grants GP 3857 and GP 6682 . 
collection of mutually exclusive $\epsilon^{\prime}$-disks $D_{1}, \cdots, D_{m}$ on $S$ which satisfy conditions (1)-(4).

The desired 2-sphere $S^{\prime}$ is obtained from $g(S)$ by pushing the disks $g\left(D_{i}\right)$ slightly into Ext $g(S)$. Specifically, for $i=1, \cdots, m$, let $B_{i}$ denote a 3 -cell in $E^{3}$ such that

(i) $g\left(D_{i}\right) \subset \mathrm{Bd} B_{i}$,

(ii) $B_{i}-g\left(D_{i}\right) \subset \operatorname{Ext} g(S)$,

(iii) $\operatorname{diam} B_{i}<3 \epsilon^{\prime}$, and

(iv) $B_{i} \cap B_{j}=\varnothing$, if $i \neq j$.

The 2-sphere $S^{\prime}=g(S) \cup\left(\cup B d B_{i}\right)-\cup g\left(\operatorname{Int} D_{i}\right)$ is homeomorphically within $\epsilon$ of $S$.

Now we show that, for a component $K$ of $C-\mathrm{Cl}(\operatorname{Int} g(S)), \mathrm{Cl} K$ meets only one of the disks $g\left(D_{i}\right)$. If $H$ denotes the unbounded component of Ext $g(S)-K$, it follows from the unicoherence of Ext $g(S)$ that $\mathrm{Bd} H$ (viewed in Ext $g(S)$ ) is connected. $\mathrm{Bd} H \subset S$, so $\mathrm{Bd} H$ is contained in one of the disks on $S$, say $D_{k}$. Then condition (4) implies that

$$
\mathrm{Cl}(\mathrm{Bd} H) \cap g(S)=\mathrm{Cl}(\mathrm{Bd} H) \cap D_{k} .
$$

Consequently, $\mathrm{Cl} K \cap g(S)=\mathrm{Cl} K \cap D_{k}$.

Let $P_{i}(i=1, \cdots, m)$ denote the sum of all components $K$ of $C-\mathrm{Cl}(\operatorname{Int} g(S))$ such that $\mathrm{Cl} K$ intersects $g\left(D_{i}\right)$. By the claim established in the preceding paragraph, $P_{i} \cap P_{j}=\varnothing$ whenever $i \neq j$. Supposing momentarily that $g(S)$ lies in $S^{3}$ instead of $E^{3}, \mathrm{Cl}(\operatorname{Ext} g(S))$ is a 3-cell, so there exist homeomorphisms $f_{i}$ from $\mathrm{Cl}(\operatorname{Ext} g(S))$ to $B_{i}$ with the additional property that $f_{i}$ is the identity on $g\left(D_{i}\right)$. Note that the diameter of each $P_{i}$ is less than $3 \epsilon^{\prime}$ and that no point of $P_{i}$ is moved as much as $\epsilon$ by $f_{i}$.

The homeomorphism $h^{\prime}$ is defined by the rule

$$
\begin{aligned}
h^{\prime}(x) & =f_{i}(x) & & \text { for } x \text { in } P_{i}, \\
& =x & & \text { for } x \text { in }\left(C-\cup P_{i}\right) .
\end{aligned}
$$

For a detailed argument establishing the theorem on the basis of this lemma, refer to Theorem 2 of [3]. The idea is to re-embed the cube with a homeomorphism $h$ which is the limit of a sequence of homeomorphisms provided by the lemma. The lemma promises a sphere for each of the homeomorphisms in the sequence, and the existence of these spheres guarantees that $h(\mathrm{Bd} C)$ can be homeomorphically approximated from $S^{3}-h(C)$. As a result of Theorem 2.1 of [1], the closure of $S^{3}-h(C)$ is a 3-cell. 


\section{REFERENCES}

1. R. H. Bing, Conditions under which a surface in $E^{3}$ is tame, Fund. Math. 47 (1959), 105-139.

2. N. Hosay, The sum of a real cube and a crumpled cube is $S^{3}$, Notices Amer. Math. Soc. 10 (1963), 666; errata 11 (1964), 152.

3. L. L. Lininger, Some results on crumpled cubes, Trans. Amer. Math. Soc. 118 (1965), 534-549.

4. F. M. Lister, Simplifying intersections of disks in Bing's side approximation theorem, Pacific J. Math. 22 (1967), 281-295.

UNIVERSITY OF TENNESSEE 